\title{
Caractérisation des systèmes de production sur les sites d'aménagements hydro-agricoles dans le doublet Dangbo-Adjohoun au sud du Bénin
}

\author{
Mouritala $\mathrm{ABOU}^{1 *}$, Ibouraïma $\mathrm{YABI}^{2}$, Isidore $\mathrm{YOLOU}^{3}$ et Euloge OGOUWALE${ }^{2}$ \\ ${ }^{1}$ Département de Géographie et Aménagement du Territoire (DGAT), FASHS, UAC, Bénin. \\ ${ }^{2}$ Laboratoire Pierre Pagney, Climat, Eau, Ecosystèmes et Développement (LACEEDE), DGAT, FASHS, \\ UAC-Bénin. \\ ${ }^{3}$ Département de Géographie et Aménagement du Territoire (DGAT), FLASH, UP, Bénin. \\ *Auteur correspondant, E-mail : elmouth.abou@yahoo.fr; Tél : (+229) 97608079
}

\section{RESUME}

La maîtrise de l'eau à des fins agricoles constitue un enjeu de taille dans les pays comme le Bénin où les besoins alimentaires sont en nette progression dans un contexte d'incertitudes climatiques. La présente recherche vise à caractériser les systèmes de production sur les aménagements hydro-agricoles dans le doublet Dangbo-Adjohoun situé dans la basse vallée de l'Ouémé. Les enquêtes de terrain ont été conduites dans huit (8) arrondissements (Késsounou, Houédomey, Gbéko, Zounguè, Gangban, Kodé, Démè et Azowlissè) auprès de 254 exploitants des sites d'aménagement hydro-agricole. Les informations qualitatives et quantitatives ont été recueillies par un questionnaire élaboré à cet effet. Le traitement des données a été fait par le test de Kruskal-Wallis pour tester la différence significative ou non entre les superficies emblavées sous les différents modes de faire valoir. Ainsi, l'analyse factorielle des correspondances simples a été utilisée pour décrire la spécificité au sein des arrondissements au regard des classes de superficie d'une part et des modes de faire valoir d'autre part. Le test d'homogénéité a permis de déterminer la tendance des systèmes de production. Les résultats d'analyse ont révélé l'existence de quatre (4) types de systèmes de production: le système de production pluviale, les systèmes de production irriguée, les systèmes de production des cultures de décrue et les systèmes de production de la pêche continentale. Pour cette recherche, le système de production pluviale qui se pratique sur le plateau n'a pas été pris en compte. Par ailleurs, les superficies aménagées varient d'un arrondissement à un autre en fonction des modes d'accès à la terre. Ainsi, l'héritage à la superficie moyenne la plus élevée, 5.78 ha suivi de propriétés collectives 1.64 ha, l'achat et l'emprunt ont respectivement les plus faibles superficies, 0.98 et 0.75 ha. Cette étude a montré les systèmes de production qui contribuent au développement des aménagements hydro-agricoles dans la plaine inondable.

(C) 2018 International Formulae Group. All rights reserved.

Mots clés : Dangbo-Adjohoun (vallée de l'Ouémé), aménagements hydro-agricoles, caractéristiques, systèmes de production agricole.

\section{Characterization of the production systems on the sites of agricultural hydro amenities in the double Dangbo-Adjohounin in the south of Benin}




\section{ABSTRACT}

The mastery of water for agricultural ends constitutes a stake of size in countries as Benin where the food needs are in clean progression in a context of climatic uncertainties. The present research aimed at characterizing the systems of production on the agricultural hydro amenities in the double DangboAdjohounlocated in the low valley of Ouémé. The investigations have been conducted in eight (8) areas (Késsounou, Houédomey, Gbéko, Zounguè, Gangban, Kodé, Démè and Azowlissè) by 254 operators of the agricultural hydro planning sites. The qualitative and quantitative information's have been collected by a questionnaire elaborated to this effect. The treatment of the data has been made by the ballast of KruskalWallis to test the meaningful difference or no between the surfaces arranged under the different fashions to put forward. Thus, the factorial analysis of the simple correspondences has been used to describe the specificity on the one hand within the areas to the look of the surface classes and of the fashions to put forward on the other hand. The test of homogeneity permitted to determine the tendency of the production systems. The results of analysis revealed the existence of four (4) types of production systems: the system of pluvial production, the systems of production irrigated the systems of production of the subsidence cultures and the systems of production of the continental fishing. For this research, the system of pluvial production that is practised on the tray has not been taken into account. Otherwise, the arranged surfaces vary from an area to another according to the fashions from access to the earth. Thus, the inheritance has the surface middle most elevated 5.78 consistent ha of collective properties 1.64 ha, the purchase and the loan have the weakest surfaces respectively 0.98 and 0.75 ha. This survey showed the systems of production that contribute to the development of the agricultural hydro amenities during the rain.

(C) 2018 International Formulae Group. All rights reserved.

Keywords: Dangbo-Adjohoun (valley of Ouémé), arrangement characteristic hydro agricultures, agricultural systems of production.

\section{INTRODUCTION}

L'agriculture constitue une ressource territoriale historique majeure qui contribue au bien-être humain en produisant, entre autres, de la nourriture, des matières premières et de l'emploi (Terrier et al., 2010). Elle contribue au développement économique des pays du tiers monde et reste en général, l'activité principale des populations. Selon la FAO (2005), 80\% de la population africaine sont rurales et dépendent directement des produits agricoles. En Afrique de l'Ouest, l'agriculture a un poids économique particulier puisque dans la zone de l'Union Economique et Monétaire Ouest Africaine (UEMOA), elle contribue pour $30 \%$ au Produit Intérieur Brut (PIB) et emploie plus de 50\% de la population active (Renard et al., 2004). Le Bénin dispose de plusieurs bas-fonds et plaines d'inondation auxquels s'intéressent davantage les paysans face à la dégradation sans précédent des terres du plateau (Agbossou et Danvi, 2000 ; Boko, 2016). Ces bas-fonds et plaines présentent des atouts incontestables pour la production agricoles dans la mesure où leur aménagement adapté permet de limiter les effets négatifs des aléas hydro-climatiques (Boraud et al., 2015). Mais, les impacts négatifs de l'exploitation incontrôlée de ces écosystèmes peuvent se traduire par une baisse des rendement s'aggravent au fur et à mesure de l'intensification du réchauffement du climat (Abdoul Habou et al., 2016). La conséquence de tous ces effets est la pénurie répétée des produits agricoles sur le marché surtout en période de soudure. Les ressources en eau du Bénin sont importantes mais les efforts pour assurer une maîtrise partielle ou totale en eau au profit des cultures sont encore minimes (AQUASTAT, 2005 ; Sintondji et al., 2013). De plus, les productions agricoles essentiellement pluviales connaîtraient des baisses de productivité de 20 à $30 \%$. Cette diminution de la productivité agricole se traduirait par une vulnérabilité sociale plus accrue (Ogouwalé, 2006). La forte variabilité 
climatique a induit la dégradation du milieu écologique, perturbé les systèmes de production, bouleversé le calendrier agricole du paysan et rendu non opérationnelles les normes culturales empiriques en vigueur chez les populations paysannes (Ogouwalé, 2004). Les activités agricoles y sont donc vulnérables face aux effets des chocs climatiques car elles sont exposées aux différentes manifestations (irrégularité des pluies, inondation, sécheresse) alors que les moyens (matériels et financiers) nécessaires à une bonne adaptation sont insuffisants. Pour rendre le potentiel d'eau disponible au profit de la population, il faut donc des efforts de mobilisation et d'aménagement permettant la gestion et l'exploitation judicieuse au profit de la production. Les exploitants agricoles des Communes de Dangbo et d'Adjohoun, développent une grande diversité de systèmes de production sur la plaine d'inondation qu'il convient de caractériser. La présente étude vise à caractériser les systèmes de production pour une amélioration du rendement dans les périmètres hydro-aménagés.

\section{MATERIEL ET METHODES}

\section{Milieu d'étude}

Situées dans le département de l'Ouémé entre $6^{\circ} 36^{\prime}$ et $6^{\circ} 43^{\prime}$ de latitude nord et entre $2^{\circ} 21^{\prime}$ et $2^{\circ} 35^{\prime}$ de longitude est, les Communes de Dangbo et d'Adjohoun (Figure 1) sont limitées au nord par la Commune de Bonou, au sud par la Commune des Aguégués, à l'est par la Commune de Sakété et à l'ouest par la Commune de Zè. Elles couvrent une superficie de $457 \quad \mathrm{~km}^{2}$ (INSAE/RGPH, 2002).

Le milieu d'étude est composé de deux unités géomorphologiques : un plateau d'altitude comprise entre 20 et $200 \mathrm{~m}$ présente des ondulations moyennes fortes et une plaine inondable ne dépassant pas $10 \mathrm{~m}$ d'altitude d'axe nord-sud qui, dans la topo-séquence estouest, jouxte le plateau. Elle s'étend de part et d'autre du fleuve Ouémé qui l'inonde annuellement entre les mois de juillet et novembre. Sur la plaine inondable, il existe des sols alluviaux et colluviaux dans le lit du cours d'eau, les vertisols dans les marécages. Ces plaines, composées d'éléments fins, sont hydromorphes en permanence ou temporairement si elles sont situées sur les bourrelets de berges (Zannou, 2016). Les sols ferrugineux tropicaux et les sols ferralitiques constituent deux autres classes caractéristiques du plateau.

L'hydrographie du milieu de recherche est composée du fleuve Ouémé qui entre ensuite dans les formations sédimentaires du bas-Bénin et coule dans les alluvions récentes qui le bordent de part et d'autre. Le fleuve Ouémé, d'une superficie de 4980 km², dispose 60000 ha de terre irrigable. Le milieu de recherche dispose environs de 5547350 ha de terre cultivable, 7466 ha de terre aménageable et 2990 ha de terre aménagée par le Projet d'Appui à la diversification Agricole (PADA) et le Programme d'Appui aux Infrastructures Agricoles dans la Vallée de l'Ouémé (PAI-VO) ainsi que les producteurs, soit $40 \%$ des terres aménageables ((Secteur Communal pour le Développement Agricole/Adjohoun, 2016).

Le climat, quant à lui, est caractérisé par un régime à quatre saisons. Il y a une grande saison des pluies de mars à juin (4 mois), une petite saison sèche de juillet à août (2 mois), une petite saison des pluies commençant en septembre et s'achevant en octobre (2 mois) et une grande saison sèche du mois de novembre à février (4 mois). Ce type de climat fait la transition entre le climat subtropical et le climat subéquatorial. Son régime, de type subéquatorial, se caractérise par une seule période de basses eaux qui dure en général sept mois, de décembre à juin et par une seule période de crue, de trois à quatre mois environs. La crue commence habituellement vers fin juillet et finit avant novembre (Lalèyè et al., 2006). Les températures moyennes mensuelles de la zone d'étude varient entre $26,7{ }^{\circ} \mathrm{C}$ (janvier) et 28,2 ${ }^{\circ} \mathrm{C}$ (mars). 


\section{Données utilisées et leurs sources}

Les données utilisées concernent les effectifs des ménages qui développent les aménagements hydro-agricoles et les superficies emblavées disponibles à la Direction Départementale de l'Agriculture, de l'élevage et de la Pêche de l'Ouémé. Les données sur l'effectif des ménages sont fournies par l'Institut National de la Statistique et de l'Analyse Economique (INSAE-Cotonou).

\section{Choix des arrondissements et des enquêtés}

Pour recueillir les informations sur les caractéristiques des systèmes de production des sites d'aménagements hydro-agricoles dans le doublet Dangbo-Adjohoun, les enquêtes ont été réalisées dans huit (08) arrondissements (Késsounou, Houédomey, Gbéko, Zounguè, Gangban, Kodé, Démè et Azowlissè) sur les quinze (15) que compte le milieu de recherche. L'échantillonnage raisonné des arrondissements a été effectué suivant les critères de proximité du fleuve Ouémé qui facilite les activités hydroagricoles sur la plaine d'inondation.

Le choix raisonné des exploitants agricoles est fait sur la base des aménagements hydro-agricoles menés par ceux-ci sur la plaine et suivant leur âge (supérieur à 20 ans).

La taille de l'échantillon a été déterminée grâce au protocole de Schwartz (1995) par la formule : $\mathrm{n}=\mathbf{Z} \boldsymbol{\alpha}^{\mathbf{2}} \mathbf{x} \mathbf{p q} / \mathbf{i}^{\mathbf{2}}$ avec : $\mathbf{Z} \boldsymbol{\alpha}=1,96$ : écart réduit correspondant à un risque $\alpha$ de $5 \% ; \mathbf{p}=\mathrm{n} / \mathrm{N}$ avec $\mathrm{p}$ la proportion des ménages qui développent les aménagements hydro-agricoles par rapport aux ménages total dans les Communes d'étude $\mathbf{i}=$ précision désirée égale à $6 \% ; \mathbf{q}=$ 1- $\mathrm{p}, \mathbf{n}=(1,96)^{2} \times \mathrm{p}(1-\mathrm{p}) / 0,05^{2}$. Au total, 254 ménages agricoles qui font les aménagements hydro-agricoles sont enquêtés dans les huit (08) arrondissements retenus (Tableau 1).

\section{Méthodes de collecte des données}

Le questionnaire administré aux exploitants agricoles a permis de réaliser les entretiens sémi-structurés et le focus-groupes. Ces entretiens sont basés sur: les modes d'acquisition des terres, les superficies emblavées, les pesticides, les fertilisants, la production, la construction des casiers, etc. La grille d'observation a permis de constater de visu les techniques d'irrigation et de drainage sur les périmètres aménagés, l'association et l'assolement des cultures maraîchères.

\section{Traitement et analyse statistique des données}

Les données collectées ont fait l'objet d'un dépouillement manuel et ont été traitées à l'aide du tableur EXCEL 2007 (le tableur a servi à tracer les Figures et Tableaux).

Les analyses ont été effectuées dans le logiciel R 3.3.2 avec le package FactoMineR pour les AFC et le package PMCMR pour Nruskal Kramer Nemenyi (Core Team, 2016). A cet effet, le test de Kruskal-Wallis a été utilisé pour évaluer l'existence de différence significative ou non entre les superficies médianes emblavées sous les différents modes de faire valoir (Gnagne et al., 2015). Des boxplots ont été réalisés suivis du test de structuration des médianes de Nruskal Kramer Nemenyi pour illustrer les modes de faire valoir qui sont similaires dans l'ensemble. L'analyse factorielle des correspondances simples a été utilisée pour décrire la spécificité au sein des arrondissements au regard des classes de superficie emblavées d'une part et au regard des modes de faire valoir d'autre part. Le test d'homogénéité (analyse des proportions) a permis de déterminer la tendance des systèmes de production sur la plaine inondable du milieu de recherche, le seuil de significativité étant de $5 \%$. 


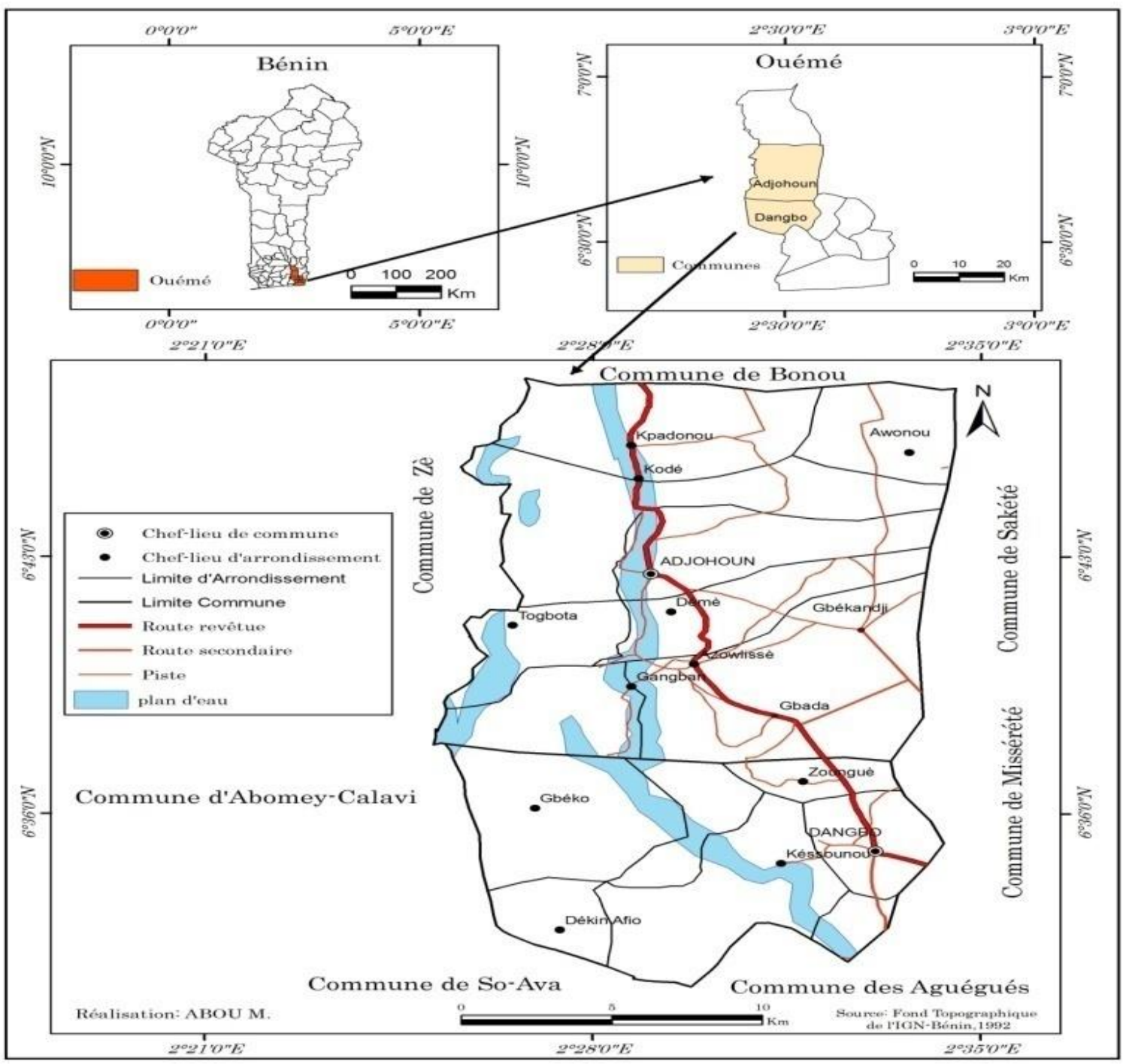

Figure 1 : Situation géographique du milieu de recherche.

Tableau 1 : Nombre des ménages enquêtés.

\begin{tabular}{cccc}
\hline Communes & Arrondissements enquêtés & Ménages gricoles/aménagement & $\begin{array}{c}\text { Nombre de ménages } \\
\text { enquêtés }\end{array}$ \\
\hline \multirow{3}{*}{ Dangbo } & Gbeko & 2777 & 33 \\
& Houedomey & 3653 & 44 \\
& Kessounou & 2870 & 34 \\
& Zounguè & 2382 & 28 \\
\hline \multirow{2}{*}{ Adjohoun } & Gangban & 3164 & 38 \\
& Kodé & 1241 & 15 \\
& Démè & 520 & 06 \\
\hline Total & Azowlissè & 4701 & 56 \\
\hline
\end{tabular}

INSAE, RGPH4 et SCDA-Dangbo/Adjohoun, 2016. 


\section{RESULTATS}

\section{Superficies emblavées et modes de faire valoir}

L'analyse de l'importance des classes de superficies suivant les modes de faire valoir a révélé que ce soit de façon globale ou au sein des classes de superficie une différence très hautement significative entre les superficies médianes des différents modes de faire valoir (Tableau 2 et Figure 2).

L'analyse du Tableau 2 et de la Figure 2 montre que de façon globale, l'héritage a la superficie médiane et moyenne la plus élevée $(5.78 \mathrm{ha})$ et diffère très significativement $(\mathrm{P}<0,001)$ de tous les autres modes de faire valoir. Il est suivi de propriétés collectives (1.64 ha). De façon globale, l'achat et l'emprunt ont les plus faibles superficies ( 0.98 et 0.75 ha respectivement) et ne sont pas significativement différents entre eux $(\mathrm{P}>0.05)$ et de même de propriété collective et $\mathrm{du}$ fermage. Les mêmes tendances sont observées au sein des classes de superficie. Ces observations sont bien illustrées par les boxplots réalisés (Figure 2).

\section{Importance des classes de superficies par arrondissement}

L'analyse de l'importance des superficies de terre entre les arrondissements du milieu de recherche à travers l'analyse factorielle des correspondances montre que les premières composantes principales expliquent à $100 \%$ toute la variabilité des superficies dans les milieux (Figure 3).

La prise en compte simultanée de la représentativité et de la contribution des classes de superficie et des arrondissements à formation des axes a révélé que la majorité des terres des ménages agricoles dans les arrondissements de Gangban, Démè et Houédomey ont une superficie comprise entre 1.5 et 3ha (Figure 3). On note aussi une proportion importante des terres de superficie comprise entre 0 et 1.5 ha à Gangban. Les arrondissements d'Azowlissè, Zounguè, Késsounou, Gbéko sont les milieux où les terres disposées par les ménages sont majoritairement comprises entre 3 ha et plus et entre 0 et 1.5 ha. C'est les terres à superficie comprise entre 0 et 1.5 ha qui sont les dominantes à Kodé suivies de 3 ha et plus.

Importance des modes de faire valoir par arrondissement

L'analyse de l'importance des modes de faire valoir des terres entre les arrondissements des milieux d'étude à travers l'analyse factorielle des correspondances montre que les premières composantes principales expliquent à $81.77 \%$ toute la variabilité des modes de faire valoir des terres dans les milieux (Figure 4).

La projection simultanée des modes de faire valoir des terres et des arrondissements tout en tenant compte de la représentativité et de la contribution des modes et des arrondissements à la formation des axes révèle que, les propriétés collectives, le fermage et l'emprunt sont les modes d'accès les plus pratiqués à Houédomey. A Azowlissè, c'est l'héritage et l'emprunt qui sont les plus pratiqués contrairement à Gbébo, Késsounou et Zounguè où c'est l'achat qui sont les modes dominants. L'héritage est aussi la forme dominante des terres acquises à Gangban et Kodé. Démè s'est révélé comme une observation supplémentaire et de ce fait ne possède rien de dominant par rapport aux autres arrondissements.

\section{Systèmes de production agricole Systèmes irrigués intensifs}

Dans les Communes de Dangbo et d'Adjohoun, les exploitants agricoles des sites d'aménagements hydro-agricoles mènent maints systèmes de production irrigués sur la plaine inondable.

\section{Fauchage et endigage}

Le fauchage et l'endigage (Planche 1) concernent essentiellement la production du riz dans les aménagements hydro-agricoles. En effet, les riziculteurs commencent par préparer les casiers dans le mois d'octobre après la crue. Le nettoyage de ces casiers est la première opération de la campagne agricole de l'année. Ils démarrent les activités par le fauchage des herbes dans l'eau. Cela favorise la décomposition des herbes dans l'eau et enrichit les casiers en sels minéraux et matières organiques (Balogoun, 2008). 
Ensuite, l'endigage, permet de délimiter les casiers à l'aide des herbes sèches. Selon les résultats du terrain, un casier mesure $25 \mathrm{~m}$ sur $25 \mathrm{~m}$. Après la construction des digues, on constate la présence de l'eau mélangée à la boue dans les casiers qui n'attendent que le repiquage.

\section{Pépinière, repiquage et entretien}

Bien avant ces activités précédentes, 97\% des riziculteurs enquêtés font la pépinière derrière leur habitation ou sur le plateau à cause de la crue qui envahit l'exploitation. A cette étape, les exploitants agricoles réalisent des billons de $1 \mathrm{~m}$ de large et $10 \mathrm{~m}$ de longueur. Ensuite, ils procèdent au nivellement ou planage du sol, puis remuent le sol et rependent les grains ou la semence. Trois jours plus tard, on arrose les grains germés jusqu'à la maturation qui dure trois semaines voire un mois.

Outre le riz, le repiquage convient également aux cultures de piment, tomate et légumes. La variété de riz mise à la disposition des riziculteurs est IR 854, qui est une variété à haut rendement et de type irrigué. Selon les résultats de terrain, deux systèmes de repiquage sont observés dans le milieu de recherche: le Système de Riziculture Intensive (SRI) et le Système Conventionnel (SC). Le Système de Riziculture Intensive (SRI), pratiqué par $25 \%$, consiste à mettre un plant par poquet, à chaque $25 \mathrm{~cm}$ selon les normes recommandées par les Secteurs Communaux du Développement Agricole (SCDA) du milieu de recherche. Selon les résultats des enquêtes de terrain, la dose moyenne de semence utilisée pour le riz IR 854 est de $10 \mathrm{~kg} / \mathrm{ha}$. Ce que les riziculteurs ne respectent pas, du coup, cette pratique affecte négativement le rendement.

Le premier désherbage se fait trois semaines environ après le repiquage. Il consiste à enlever les mauvaises herbes qui peuvent empêcher la croissance des plants dans les casiers. Et le deuxième désherbage au début du montaison (période des fleurs) pour éviter la chute des fleurs (Balogoun, 2008).

\section{Irrigation, drainage et récolte}

Dans le but de satisfaire les besoins hydriques de la culture riz, les riziculteurs enquêtés pratiquent l'irrigation par pompage, basée sur l'utilisation de motopompe, des tuyaux et les eaux du canal de dérivation (Planche 2). Cet aménagement, pratiqué par 93\% des exploitants agricoles enquêtés, est basé sur le système de casierage, une séparation de l'exploitation par des diguettes et un planage des casiers. La régulation de l'eau est assurée grâce à des tuyaux en plastique qui permettent le remplissage et la vidange des casiers.

Toutefois, on observe des cultures sous irrigation par gravitaire ou drainage, qui repose généralement sur un drain central permettant d'évacuer l'eau ou d'approvisionner l'eau selon les cas. Ce système qui nécessite la disponibilité de l'eau et un relief approprié se pratique sur les cultures maraîchères (gombo, piment, tomate et légumes). Les drains creusés selon le sens de la pente sont souvent parallèles aux cultures, ce qui facilite rapidement l'écoulement de l'eau en cas d'inondation.

Aussi, l'opération de récolte se fait généralement de façon manuelle et nécessite une main d'œuvre abondante à cause du temps réservé à cet effet. Selon les résultats de terrain, $98 \%$ des membres de la famille interviennent dans la récolte surtout les femmes et les enfants. Les riziculteurs font une récolte qui démarre vers la fin de la première saison entre février et mars. Pour $90 \%$ des riziculteurs enquêtés, la récolte du riz se fait à l'aide d'un couteau pour couper les tiges puis les mettre en tas. Après la récolte, les riziculteurs quantifient les rendements obtenus par tonneau, kilogramme, tonnage ou par nombre de sacs de $50 \mathrm{~kg}$. Pour le Secteur Communal de Développement d'Adjohoun, un sac du riz de $50 \mathrm{~kg}$ repiqué donne à la récolte huit(08) sacs de $50 \mathrm{~kg}$ par casier. Selon les résultats du terrain, $25 \%$ du riz récoltés sont destinés à l'autoconsommation puis le reste est vendu sur le marché local ou à la Coopération d'Appui à la filière Rizicole de l'Ouémé-Plateau (CAFROP). Dans le but de maximiser les rendements et de garantir l'autosuffisante alimentaire, les riziculteurs s'entendent et s'organisent en association ou groupement. 


\section{Système de cultures de décrue}

On rencontre également ce système en zone agricole dans la plaine inondable du milieu d'étude en bordure des aménagements sous irrigation. Les unités de production sont de type familial, individuel ou association avec une moyenne de 4 à 5 personnes par exploitation. La taille des exploitations varie d'un individu à un autre et d'une association à une autre.

\section{Préparation du sol, semis et entretiens}

Après la crue, les activités agricoles démarrent par le défrichage qui nécessite l'utilisation de la houe ordinaire appelé en langue locale «adjaba» qui est un coupecoupe en forme de demi-cercle fabriqué à cet effet et permet d'apprêter le terrain avant le semis.

Les exploitants agricoles préparent le sol en creusant des poquets de 10 à $15 \mathrm{~cm}$ de profondeur à l'aide d'un bâton, ce qui est propre aux sols fertiles et n'associe aucune culture ni facilite la décomposition de façon rapide de la graine mise sous terre. Mais, ces herbes sèches, donnent de l'ombrage et de l'humidité au sol. A la différence de repiquage, le semis est pratiqué sur le maïs, le haricot blanc et le gombo. Environ 57\% des exploitants agricoles enquêtés de la plaine inondable pratiquent également le buttage pour les cultures de tubercules comme la patate douce et la plante à racine, le manioc.

Par ailleurs, pour la préparation du sol, l'entretien des plants et la conservation des grains, les exploitants agricoles enquêtés utilisent les pesticides. Il s'agit des insecticides, les herbicides, les fongicides, les nématoïdes. En effet, les herbicides sont pulvérisés sur les herbes afin de les rendre sèches avant le semis ou le repiquage des plants. Fréquemment, les insecticides sont utilisés pour le traitement des plants jusqu'à la récolte à cause des insectes et rats dévastateurs.

\section{Fertilisation et main d'ouvre agricole}

L'urée et le NPK subventionnés par l'Etat à 12000 FCFA, sont des engrais chimiques utilisés dans les arrondissements de Houédomey et d'Azowlissè. On constate parallèlement que ces produits phytosanitaires sont approvisionnés sur le marché informel venant d'horizons diverses : $18 \%$ au Centre d'Action Régionale pour le Développement Rural (CARDER), 62\% s'approvisionnent sur le marché local et $20 \%$ au Nigéria. L'application de ces produits ne réponde pas aux normes recommandées, ce qui expose l'environnement à des risques très élevés.

Dans cette catégorie de système, presque tous les modes d'accès à la terre sont pratiqués par les exploitants agricoles (héritage, achat, emprunt, fermage et la propriété collective). Mais, l'héritage est le plus dominant, ce qui permet à $60 \%$ d'héritiers d'emblaver de grandes superficies allant de 1,5 à 3 ha et plus. Sur lesquelles on pratique la monoculture (maïs, gombo), les cultures associées (niébé et la tomate associée au piment et légumes), l'assolement (maïs, légumes, piment, tomate et niébé) et la rotation des cultures.

A Dangbo comme Adjohoun, les ménages agricoles enquêtés sont constitués des hommes, femmes et leurs enfants qui organisent les activités agricoles. On y dénombre la main d'œuvre salariée, familiale, les groupes d'entraide et autres (Figure 5).

La Figure 5 montre que la main d'œuvre salariée estimée à $51 \%$ est plus dominante, ensuite viennent la main d'œuvre familiale $44 \%$, les groupes d'entraide $4 \%$ et autres $1 \%$. La proportion impressionnante de la main d'œuvre salariée s'explique par la présence des ouvriers appelés en langue locale «kpaka» de la préparation du sol jusqu'à la transformation des produits. Le Tableau 3 présente les activités de l'ouvrier, la durée de travail et la rémunération.

L'analyse du Tableau 3 présente la durée journalière de l'exécution des activités agricoles et le coût de l'opération. Ainsi, un ouvrier qui défriche de $7 \mathrm{~h}-12 \mathrm{~h}$ ou $7 \mathrm{~h}-18 \mathrm{~h}$ gagne respectivement 1500 FCFA ou 2000 FCFA, 4000 FCFA ou 5000 FCFA. Pendant la construction des digues, l'ouvrier qui réalise un casier est rémunéré à 7000 FCFA ou 8000 FCFA. Par contre, lors du sarclage et le buttage, il prend 2000 FCFA. De $7 \mathrm{~h}-18 \mathrm{~h}$, 
pendant le semis ou repiquage et la récolte, il est rémunéré à 3000 FCFA.

De tout ce qui précède, à la différence du riz, les autres cultures bénéficient de deux saisons. En effet, la première saison démarre d'octobre à février. Par contre, la seconde saison s'impose entre mars et mai avec la préparation du sol en mars puis le semis (maïs, gombo, niébé etc.) ou repiquage (légumes, piment, tomate) et l'entretien dans le même mois ensuite suivront les récoltes en mai. La plaine inondable étant riche, ce système permet de maximiser les rendements afin de garantir l'autosuffisance alimentaire.

\section{Systèmes piscicole et pêche}

Le Bénin est caractérisé par d'innombrables vallées et de vastes plaines d'inondations favorables aussi bien à la pisciculture extensive, semi-intensive qu'intégrée (Imorou Toko, 2011). En effet, dans la zone de recherche, les producteurs piscicoles utilisent plusieurs systèmes de production sur la plaine inondable. La pêche dans cette zone représente 30 à $40 \%$ du revenu de paysan, ce qui est considérable (Chikou, 2006). En effet, des techniques piscicoles identifiées concernent les trous traditionnels à poissons, des étangs, des enclos et des cages. Toutefois, les pêcheurs utilisent les techniques de filet, des hameçons, les nasses à poisson permettent de capturer les poissons.

\section{Pêche de filet et trous à poisson}

En dehors de la pêche ordinaire avec l'hameçon, les nasses, les "acadjas", la pêche de filet et les trous à poissons (Planche 3) occupent une proportion plus élevée $28 \%$ dans le département de l'Ouémé (Gouvernement de la République du Bénin, 2009).En effet, c'est le type de filet le plus employé dans la plupart des eaux intérieures béninoises. Il s'agit d'une nappe de filet tronconique, lestée de plombs sur le pourtour de la base, munie d'une corde de manipulation et de rappel au sommet, que le pêcheur jette en parachute pour coiffer les poissons depuis la berge (Cakpo-kiossa, 2012).

Toutefois, on observe dans la zone de recherche, deux catégories de trous à poisson dont la première appelée en langue locale «houèdo » est une tranchée creusée dans la plaine d'inondation des fleuves et lacs du Sud-Bénin et dans lesquelles les poissons restent emprisonnées après le retrait des eaux de crue (Elègbè et al., 2015). Pour la réaliser, elle nécessite une main d'œuvre salariée qui varie entre 2000 à 3000 FCFA le mètre cube. La deuxième catégorie dénommée «ahlô » est également une tranchée creusée dans les basses parties de la plaine d'inondation un peu éloignée des cours d'eau. Ils sont confectionnés à 1000 FCFA le mètre cube.

Les Photos 3.1 et 3.2 de la Planche 3 montre respectivement la pêche de filet et un trou à poisson à Gangban. Cependant, il n'est pas rare d'observer des trous à poisson de forme rectangulaire, carré et circulaire de dimensions variées, d'une profondeur comprise entre 1,85 à $1,95 \mathrm{~m}$ et des superficies inférieures à $500 \mathrm{~m}^{2}$ et d'autres comprises entre 500 et $1000 \mathrm{~m}^{2}$. Ainsi, pendant la crue, le fleuve Ouémé draine les différentes espèces de poissons dans ces trous creusés à cet effet. Vu la profondeur des trous, ces poissons sont souvent incapables de remonter à la surface de l'eau. En définitive, les trous à poisson constituent les techniques piscicoles la plus utilisée à Dangbo et Adjohoun. Selon le Secteur Communal du Développement Agricole (SCDA) du milieu de recherche, 220 trous à poisson ont été creusés à la main par les producteurs enquêtés répartis sur une superficie de près 8,5 ha. Pendant le retrait de l'eau, ces trous sont partiellement vidés, ce qui facilite la récolte à travers le filet placé tout au long de la largeur et tiré dans le sens de la longueur. Cette technique qui permet de faire des mouvements d'aller et retour nécessite une main d'œuvre abondante (familiale et salariée).

\section{Acadja, étangs et enclos}

L'acadja est une pêcherie traditionnelle largement pratiquée dans les lagunes côtières du Bénin (Sogansa, 2014). 
En effet, les "acadjas" sont construits à l'aide des branchages à l'intérieur des eaux de la plaine inondable formant des clôtures rondes à la surface de l'eau. Ainsi, ces exploitants alimentent ces clôtures en matières végétales (feuilles, branchages) qui ont la propriété de vite se décomposer tout en favorisant la multiplication des micro-organismes dont se nourrissent les poissons. Ces branchages constituent à la fois une source de nourriture pour les poissons mais facilitent également leur reproduction. Ainsi, il a été identifié de petits "acadjas" installés dans l'eau dont les rendements varient de $0,5 \mathrm{t} / \mathrm{ha} / \mathrm{an}$ à 25 t/ha/an.

Les observations directes en milieu d'étude ont permis de caractériser les étangs. On entend par étang d'inondation un étang dont la mise en charge est assurée par les crues (débordement des eaux) des cours d'eau (Mfossa, 2007). Ces étangs sont alimentés en eau par la crue causée par les eaux de ruissellement. En effet, les producteurs confectionnent des étangs traditionnels qui varient de 3 à $5 \mathrm{~m}$ de largeur sur $10 \mathrm{~m}$ de longueur et d'un mètre de profondeur.

Hormis ces étangs, $10 \%$ des producteurs fabriquent des enclos et des cages à l'aide des bambous et des filets de pêche afin d'élever les poissons et d'intensifier leur production.

Cette activité de pisciculture est souvent pratiquée par les wémènou $(92 \%)$ et les fons ( $8 \%)$. Outre l'héritage $(70 \%)$, ces producteurs enquêtés de façon individuelle, ont souvent accès aux terres piscicoles par la propriété collective (24\%) et l'achat $(6 \%)$. A la différence des associations ou groupements, l'héritage, le fermage et l'emprunt sont les principaux modes d'accès aux terres piscicoles. La pêche et la pisciculture révèlent un atout prépondérant pour les exploitants agricoles de la plaine inondable du milieu de recherche. En effet, la pisciculture paysanne apparaît comme un enjeu de développement agricole et de la sécurité alimentaire permettant de satisfaire les besoins halieutiques locaux. Elle valorise les ressources de production et participe également à la lutte contre la pauvreté.

Il faut noter qu'étant reconnu deuxième activité économique, la pêche est confrontée de nos jours à des difficultés énormes. Sa rentabilité n'est plus évidente. De surcroît, les revenus tirés de l'activité sont maigres et ne peuvent constituer une source de motivation des producteurs (Ndour et al., 2017). En effet, le nonrespect de la réglementation en matière de pêche, la mauvaise gestion des plans d'eau, l'utilisation des engins prohibés contribuent à l'appauvrissement des cours d'eau. Vu le développement des activités hydroaménagées sur la plaine d'inondation, les pêcheurs sont contraints de reconvertir pour un temps leurs activités comme ouvriers agricoles.

Tableau 2: Superficie moyenne de terre par mode de faire valoir de façon globale et suivant les classes de superficies emblavées: résultats du test de Kruskal-Wallis

\begin{tabular}{lcccccccc}
\hline \multirow{2}{*}{ MFV } & \multicolumn{2}{c}{$[\mathbf{0 - 1 , 5 h a ]}$} & \multicolumn{2}{c}{$[\mathbf{1 , 5 - 3 h a ]}$} & \multicolumn{2}{c}{ [3ha et plus } & \multicolumn{2}{c}{ Global } \\
\cline { 2 - 9 } & $\mathbf{m}$ & $\mathbf{c v}$ & $\mathbf{~ m}$ & $\mathbf{c v}$ & $\mathbf{~ m}$ & $\mathbf{c v}$ & $\mathbf{~ m}$ & $\mathbf{c v}$ \\
\hline Achat & $0.41^{\mathrm{b}}$ & 89.78 & $0.25^{\mathrm{b}}$ & 225.32 & $0.32^{\mathrm{b}}$ & 295.51 & $0.98^{\mathrm{b}}$ & 131.59 \\
Emprunt & $0.25^{\mathrm{b}}$ & 115.47 & $0.50^{\mathrm{b}}$ & 200.00 & $0.00^{\mathrm{c}}$ & 0.00 & $0.75^{\mathrm{b}}$ & 115.47 \\
Fermage & $0.31^{\mathrm{b}}$ & 141.33 & $0.35^{\mathrm{b}}$ & 245.87 & $0.69^{\mathrm{b}}$ & 190.03 & $1.35^{\mathrm{b}}$ & 141.75 \\
Héritage & $0.82^{\mathrm{a}}$ & 164.51 & $1.60^{\mathrm{a}}$ & 72.13 & $3.36^{\mathrm{a}}$ & 74.42 & $5.78^{\mathrm{a}}$ & 49.74 \\
P.Collective & $0.43^{\mathrm{b}}$ & 117.59 & $0.43^{\mathrm{b}}$ & 195.00 & $0.79^{\mathrm{b}}$ & 176.99 & $1.64^{\mathrm{b}}$ & 96.90 \\
\hline Probabilité & 0,000 & - & 0,000 & - & 0,000 & - & 0,000 & - \\
\hline
\end{tabular}

Les moyennes ayant les mêmes lettres en colonne ne sont pas significativement différentes, $\mathrm{m}=$ moyenne ; cv = coefficient de variation 


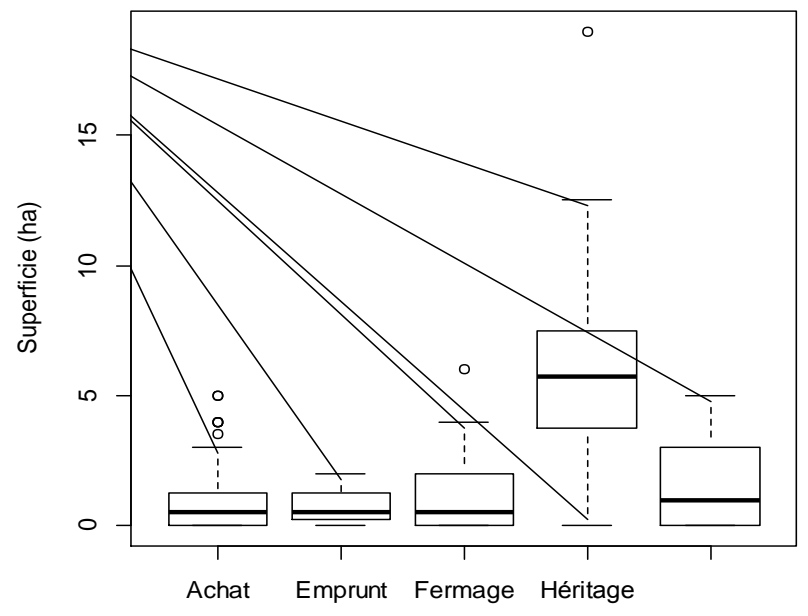

Modes de Faire Valoir

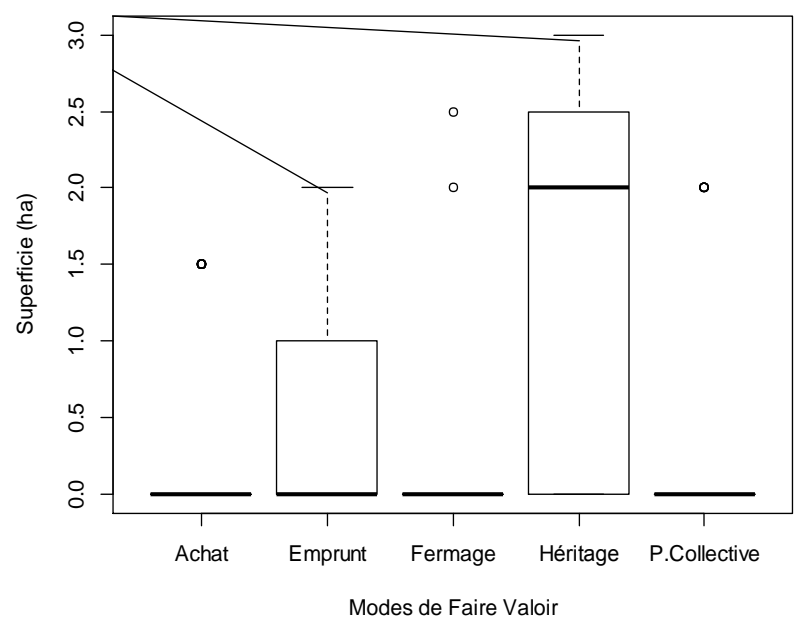

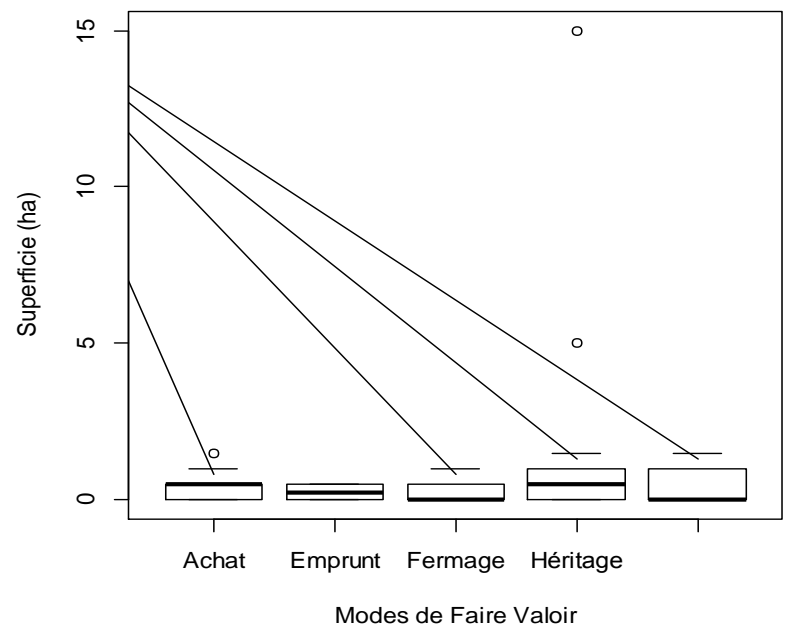

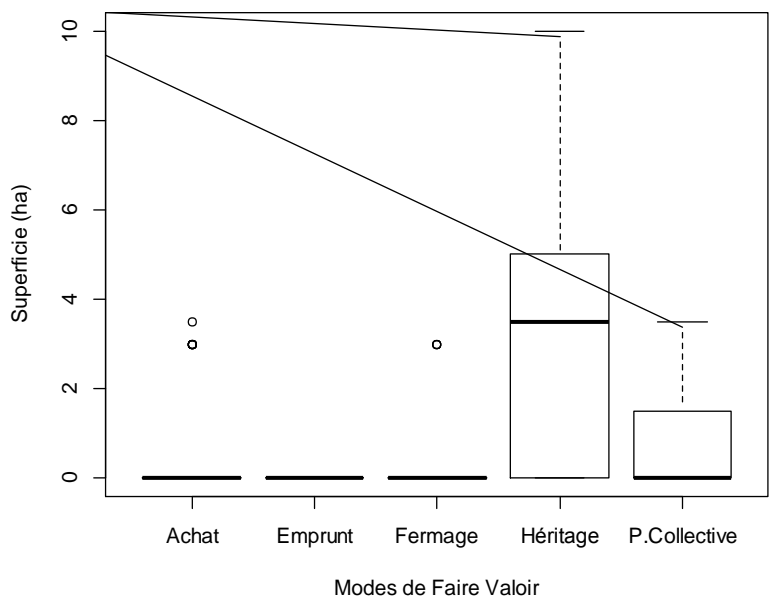

Figure 2. Modes de faire valoir au sein des classes de superficie de façon globale.

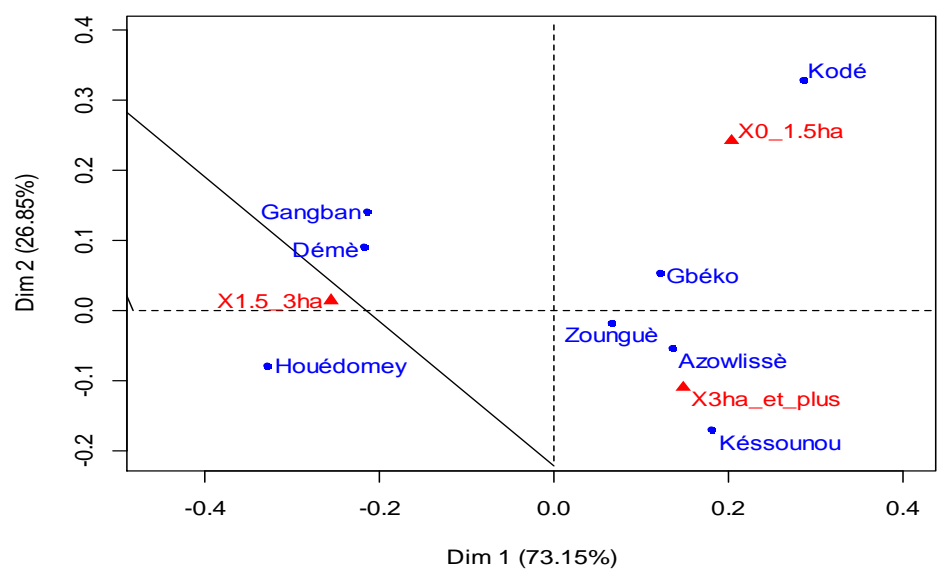

Figure 3 : Projection des classes de superficie et des arrondissements dans le plan d'axe factoriel formé par les deux premières composantes principales. 


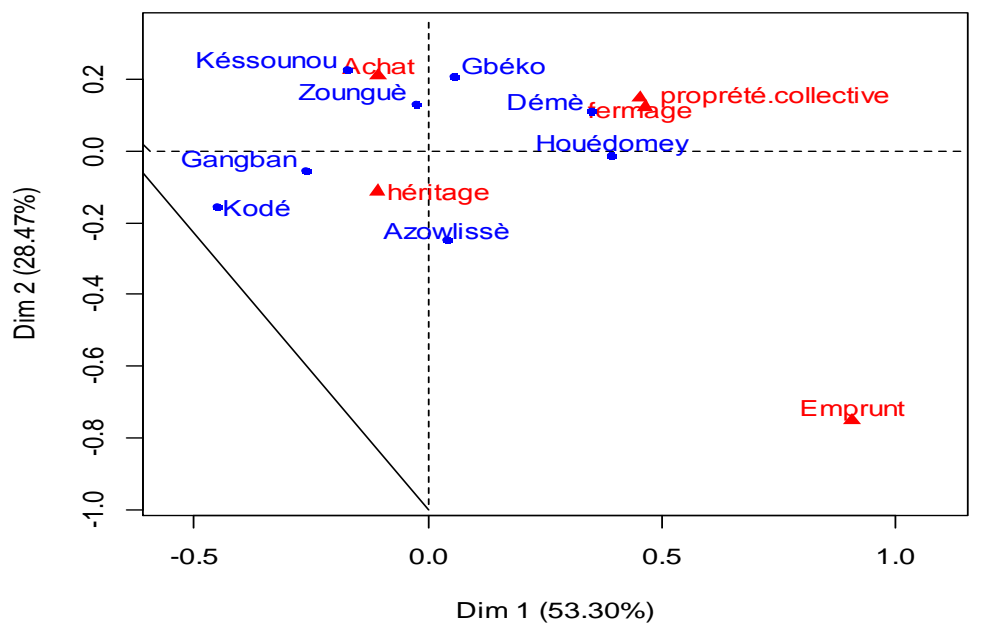

Figure 4 : Projection des modes de faire valoir et les arrondissements dans le plan d'axe factoriel formé par les deux premières composantes principales.
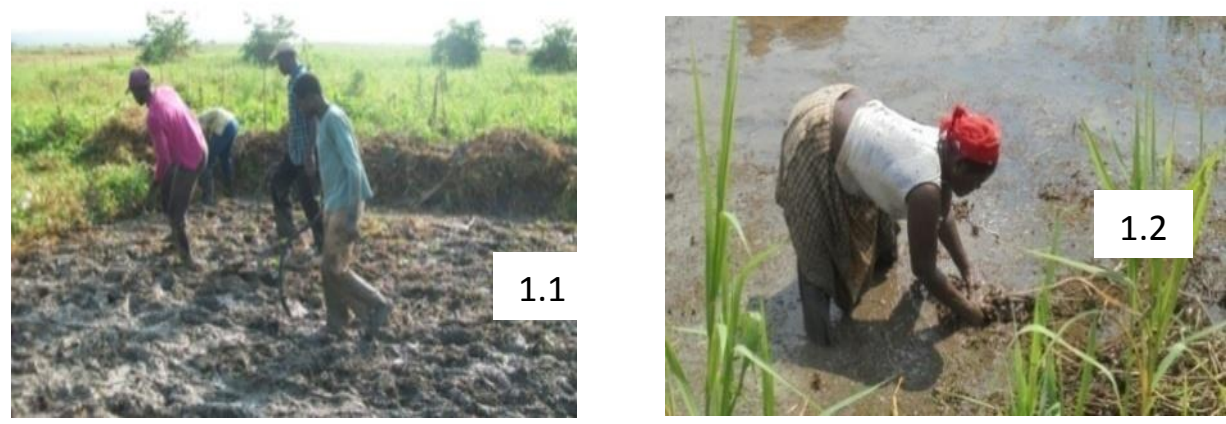

Planche 1 : Endigage à Dannou (1.1) et fauchage à Houèda (1.2).

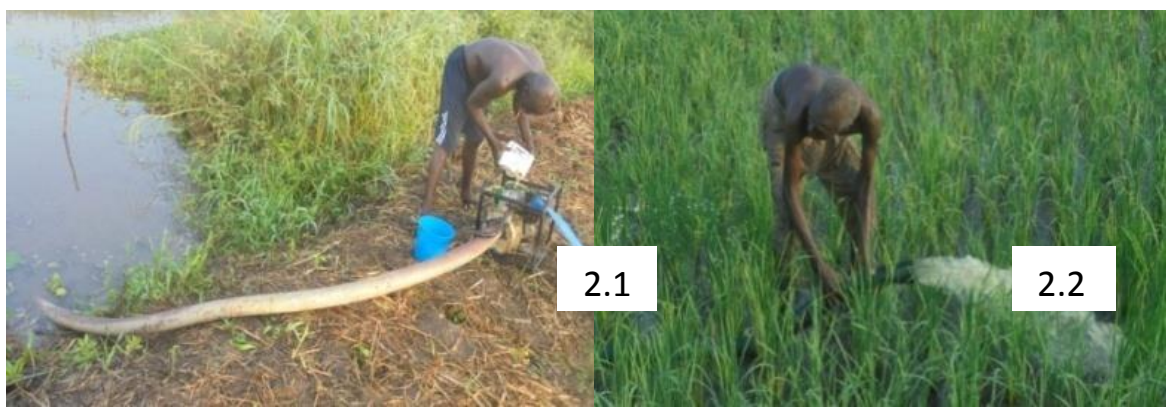

Planche 2: Motopompe (2.1) et apport d'eau du canal de dérivation sur un champ du riz par un tuyau en plastique à Houèdà (2.2). 


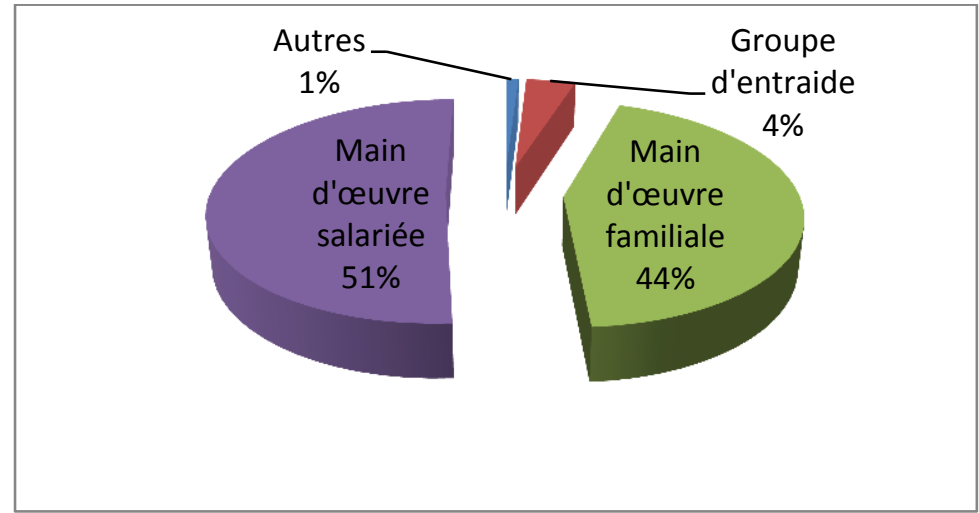

Figure 5 : Mains d'œuvre à Dangbo et à Adjohoun.

Tableau 3 : Temps mis et coût (en FCFA) de l'opération agricole.

\begin{tabular}{lcc}
\hline Activités agricoles & Temps mis/jour & Coût de l'opération \\
\hline Défrichement & $7 \mathrm{~h}-12 \mathrm{~h}$ & 1500 ou 2000 \\
& $7 \mathrm{~h}-18 \mathrm{~h}$ & 4000 ou 5000 \\
Pépinière & $7 \mathrm{~h}-18 \mathrm{~h}$ & 3000 ou 4000 \\
Construction des digues & 1 casier & 7000 ou 8000 \\
Labour/buttage & $7 \mathrm{~h}-18 \mathrm{~h} 45$ lignes de buttes & 2000 \\
Sarclage & $7 \mathrm{~h}-12 \mathrm{~h}$ & 2000 \\
& $7 \mathrm{~h}-13 \mathrm{~h}$ & 2000 \\
Semis/repiquage & $7 \mathrm{~h}-18 \mathrm{~h}$ & 3000 \\
Récolte & $7 \mathrm{~h}-18 \mathrm{~h}$ & 3000 \\
& 1 panier de piment & 1000 ou 1500 \\
Transport & 1 sac de maïs & 200 ou 300 \\
& 1 sac de piment & 100 ou 200 \\
\hline
\end{tabular}

Résultats de terrain, décembre 2016.
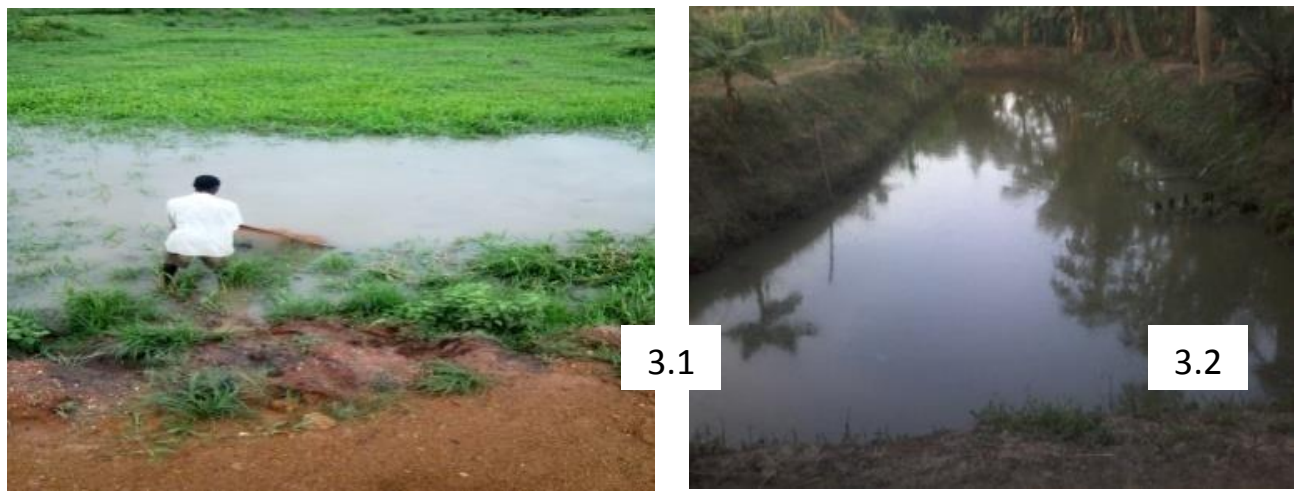

Planche 3 : Pêche de filet (3.1) et trous à poisson (3.2) à Gangban. 


\section{DISCUSSION}

\section{Modes de faire valoir et superficies emblavées}

La terre, capital foncier, est une composante importante de l'exploitation agricole. Son accès passe nécessairement par des règles préalablement définies entre une personne ou groupe d'individus (acquéreurs) et une famille ou collectivité. Au total, cinq (05) modes d'accès à la terre ont été enregistrés dans la zone de recherche. L'héritage foncier se transfère de génération en génération des terres et leurs ressources. Les transactions foncières plus monétarisées continuent entre les propriétaires terriens et les acheteurs. Ce mode d'acquisition de terre garantit une grande sécurité aux exploitants agricoles puisque le contrat de vente s'établit entre les parties contractantes et les témoins. Cette vente ne se fait pas à l'hectare mais par carré $(25 \mathrm{~m}$ sur $25 \mathrm{~m})$ et suivant certains critères : un prix moins coûteux, le rendement plus élevé après la récolte et sa proximité de l'eau. Une fois les critères remplis, les prix d'un carré oscillent entre 120.000 FCFA à 140.000 FCFA. Dans la zone de recherche, près de 1345 ha de terres sont irriguées dont la taille des exploitations est constituée de petite superficie (0-1,5 ha), de moyenne superficie $(1,5$ à $3 \mathrm{ha})$ et de grande superficie ( 3 ha et plus). Ces résultats sont presque conformes aux travaux de Yoda (2009) réalisés au Burkina Faso qui ont montré que les exploitants agricoles ruraux accèdent souvent à la terre par maints modes. Pour l'auteur, ces modes d'accès apparaissent comme une condition essentielle pour juguler la crise alimentaire actuelle et impulser le développement durable. Les terres de la propriété collective appartiennent à une collectivité, une famille ou un groupement d'exploitant. Mais, le locataire de terre a l'obligation de verser annuellement une redevance ou une partie de la récolte au propriétaire. Il doit rigoureusement respecter les clauses qui interdisent parfois au fermier de cultiver les espèces pérennes sur la parcelle. Toutefois, il est important de signaler que les résultats de cette recherche sont un peu différents à ceux de Son et al., (2016). A la différence, les modes «Achat» et « Héritage » ont disparu dans ses résultats.

\section{Systèmes de production}

Ce système de production agricole bien structuré se repose sur des moyens de production (eau, travail, terre, équipement) combinés entre eux pour assurer une production végétale et/ou animale en vue de satisfaire les objectifs et besoins de l'exploitant (ou du chef de l'unité de production) et de sa famille. D'une manière générale, les activités hydro-agricoles ne sont pas mécanisées sur la plaine inondable. Tous les travaux sont manuels. Le matériel se résume à la houe en forme de demi-cercle, les paniers, les tuyaux en plastique, le groupe électrogène (motopompe), les filets de pêche, le râteau, l'arrosoir, etc. Le manque de moyens financiers amène au moins $90 \%$ des ménages agricoles à ne pas s'investir dans le matériel. En conséquence, les opérations culturales prennent du temps par rapport à la durée prévue. La fertilisation se résume sur les engrais chimiques (l'urée et le NPK). Les insecticides (Clofos de 20\% Ec ; Lama Finer $2,5 \mathrm{Ec}$; Termicot 20\% Ec) et herbicides totaux (Forge Up; Sharp; Herbeextra et Glycel) sont utilisés couramment sur le maïs, le haricot et les cultures maraîchères. Ces résultats corroborent à ceux de la République du Sénégal (2007) sur la caractérisation des systèmes de production. Mais, ses résultats ont été élargis sur les systèmes de production arboriculture fruitière et pastoraux extensifs, qui ne se rencontrent pas dans le milieu de recherche. Dans l'ensemble, les aménagements hydro-agricoles sont sommaires (traditionnel). Le système de culture de décrue est basé sur le casierage définitif ou la maîtrise de l'eau est vraiment aléatoire. L'irrigation par pompage à l'aide d'une motopompe apporte l'eau dans les casiers rizicoles. Cette alimentation en eau vient des bâches d'eau creusées à cet effet. En période d'inondation, les drains évacuent l'eau de l'exploitation vers le canal principal. Ce constat est observé dans le Sahel ouestafricain par Barbier et al. (2011). 
La pêche continentale se pratique individuellement ou par groupement ou association. On dénombre près de 45 groupements ou associations des pêcheurs dans la zone de recherche. La pêche est une activité artisanale bien enracinée dans l'économie vivrière. Elle contribue au ralentissement de l'exode rural. Les techniques de captures restent traditionnelles et ne répondent pas aux normes recommandées par les (SCDA) du milieu de recherche. Conséquemment, la pêche continentale rencontre des difficultés qui freinent son évolution. Elles sont d'ordre technico-économique et organisationnel. L'absence de prise de conscience des pêcheurs de l'urgence d'opérer des pratiques de pêche responsable pour une exploitation durable, difficulté d'accès au crédit pour la réalisation des aménagements appropriés et problèmes de marché et de prix. Les systèmes de production de la pêche et de la pisciculture sont conformes à ceux trouvés par Sènouvo (2000). Cet auteur a identifié dans la Commune d'Adjohoun, plus précisément à Gangban, les systèmes de production de pêche comme les différents trous à poisson, les filets et les nasses. Ces résultats ont été également confirmés par les recherches réalisées par Toko (2007). Dans ces travaux, il a montré l'importance et les types de trous à poisson ainsi que les conditions d'amélioration de la production halieutique dans la vallée de l’Ouémé.

\section{Conclusion}

$\mathrm{Au}$ terme de cette recherche, on retient que la diversité biophysique des Communes de Dangbo et d'Adjohoun a permis l'occupation et l'utilisation des éléments pédologiques qu'on rencontre dans les zones agro-écologiques. De ce fait, les exploitants agricoles emblavent les superficies suivant les différents modes d'accès à la terre. Ainsi, l'héritage a la superficie moyenne la plus élevée 5.78 ha, suivi de propriétés collectives 1.64 ha, l'achat et l'emprunt ont respectivement les plus faibles superficies 0.98 et 0.75 ha. Sur ces superficies emblavées, les caractéristiques des zones agro-écologiques permettent d'observer les principaux systèmes de production pratiqués par les exploitants agricoles. Ainsi, sur la plaine inondable, les sols hydromorphes prospèrent les systèmes de cultures irriguées, les systèmes de production des cultures de décrue sont dominants et le fleuve Ouémé contribue au développement du système de production de la pêche continentale. Mais, plusieurs facteurs entravent la rentabilité de ces systèmes de production dans le milieu de recherche. Par ailleurs, avec les effets de l'inondation et de la sécheresse répétés, des orientations politiques et de l'Etat à travers des solutions concrètes aux perturbations climatiques doivent renforcer les systèmes de production existants par des actions novatrices.

\section{CONFLIT D'INTERETS}

Tous les auteurs déclarent à l'unanimité qu'il n'existe aucun conflit d'intérêts entre eux relatif à cet article.

\section{CONTRIBUTIONS DES AUTEURS}

MA a procédé à la rédaction du protocole, a conduit les travaux de terrain ainsi que l'analyse statistique des données et a écrit le manuscrit. IY a participé à la formulation de la thématique, la lecture à plusieurs reprises, la correction et l'identification de la revue pour la soumission du manuscrit. IY a participé aux enquêtes de terrain et aux analyses statistiques des données. EO a donné des orientations méthodologiques et a contribué à la lecture et la correction de ce manuscrit.

\section{REFERENCES}

Abdoul Habou Z, Boucar MK, Adam T. 2016. Les systèmes de productions agricoles du Niger face au changement climatique : défis et perspectives. Int. J. Biol. Chem. Sci., 10(3): $1262-1272 . \quad$ DOI: http://dx.doi.org/10.4314/ijbcs.v10i3. 
28

Agbossou KE, Danvi C. 2000. Diagnostic Rapide de Pré-aménagement. Rapport Provisoire. UNC/CBF : Cotonou (Bénin), 15.

AQUASTAT. 2005. L'irrigation en Afrique en chiffres. Rapport de l'enquête, 93p.

Balogoun B. 2008. Etats des lieux et perspectives de la production du riz dans la Commune de Dangbo. Mémoire de Maîtrise en Géographie, Faculté des Lettres, Arts et Sciences Humaine, Université d'Abomey-Calavi, 81p.

Barbier B, Ouédraogo H, Dembele Y, Yacouba H, Barry B, Yamin JY. 2011: L'agriculture irriguée dans le Sahel oustafricain. Cah Agric., 20: 24-33. DOI : 10.1684/agr 2011.047

BokoCSA. 2016. Caractérisation agro-sociosanitaire des bas-fonds de la Commune de Zagnanado (Département du ZOU, Bénin). Mémoire de Master, Faculté des Sciences Agronomiques, Université d'Abomey-Calavi, 106p.

Boraud NKM, Kouamé KF, Kla D. 2015. Impact des pratiques de gestion des adventices sur le rendement du riz aucentre de la Côte d'Ivoire. Int. J. Biol. Chem. Sci., 9(3): 1220-1228. DOI : http://dx.doi.org/10.4314/ijbcs.v9i3.7

Cakpo Kiossa C. 2012. Biodiversité et exploitation de quelques espèces de poissons de la rivière Sô au Bénin. Mémoire de Master Recherche, Institut de Développement Rural, Université de Bobo-Dioulasso, 113p.

Chikou A. 2006. Etude de la démographie et de l'exploitation halieutique de six espèces de poissons chats (Teleostei, Siluriformes) dans le delta de l'Ouémé au Bénin. Thèse de doctorat, Université de Liège, p. 459.

Core TR. 2016. A Language and Environment for Statistical Computing. R Foundation for Statistical Computing, Vienna, Austria. https://www.R-project.org

Elègbè HA, Imorou Toko I, Agbohessi $\mathrm{P}$, Ble C, Banag A, Chikou A, Eyango Tomedi M, Lalèyè P. 2015.Co-culture Clarias gariepinus-Oreochromis niloticus
Quels Avantages pour l'Amélioration des Performances Zootechniques et économiques des poissons élevés dans les « whedos » du delta de l'Ouémé au Bénin ? Int. J. Biol. Chem. Sci., 9(4): 1937-1949. DOI http://dx.doi.org/10.4314/ijbcs.v9i4.19

FAO (United Nations Food and Agriculture Organization). 2005. Rapport du Directeur Général sur la sécurité alimentaire, Rome, 33p.

Gnagne YA, Yapo BO, Meite L, Kouame VK, Gadji AA, Mambo V, Houenou P. 2015. Caractérisation physico-chimique et bactériologique des eaux usées brutes du réseau d'égout de la ville d'Abidjan. Int. J. Biol. Chem. Sci., 9(2): 1065-1076. DOI: http://dx.doi.org/10.4314/ijbcs.v9i2.44

Gouvernement de la République du Bénin. 2009. Rapport national sur l'Analyse Globale de la Vulnérabilité, de la Sécurité Alimentaire et de la Nutrition (AGVSAN), 154p.

Imorou Toko I. 2007. Amélioration de la production halieutique des trous à poisson (houèdos) du delta de l'Ouémé au sud bénin par la promotion de l'élevage des poissons chats. Thèse de doctorat. Facultés Universitaires notre Dame de la paix, Université de Namur, Belgique, 186p.

Imorou Toko I, Attakpa EY, Baco MN, Gouda AI. 2011. Analyse des systèmes piscicoles dans la Vallée du Niger (Nord Bénin). Int. J. Biol. Chem. Sci., 5(5): 1993-2003.

INSAE (Institut National de la Statistique et de l'Analyse Economique). 2002. Troisième Recensement de la Population et de l'Habitation. Rapport général, Cotonou, Bénin, 230p.

Lalèyé P, Chikou A, Gnohossou P, Vanderwalle P, Philipart J-C, Tengels GG. 2006. Studies on the biology of two species of catfish synodontis schall and synodontis nigrita (ostariophyi: Mochokidae) from the Ouémé River. Bénin. Belgian Journal of Zoology, 136(2): 193-201. 
Mfossa MD. 2007. Caractérisation des étangs d'inondation de la plaine des Mbô et analyse des facteurs influençant leur production piscicole. Mémoire d'Ingénieur des Eaux, Forêts et Chasses. FASAl, Université de Dschang, 75p

Ndour N, Sambou B, Diadhiou H, Sambou H, Dasylva M. 2017. Atouts et contraintes de la pisciculture traditionnelle de Bandial (Casamance, Sénégal). Int. J. Biol. Chem. Sci., 11(4): 1685-1705. DOI: http://dx.doi.org/10.4314/ijbcs.v11i4.22

Ogouwalé E. 2004. Changements climatiques et sécurité alimentaire dans le Bénin méridionale. Mémoire de DEA, UAC/EDP/FLASH, 119p.

Ogouwalé E. 2006. Changements climatiques dans le Bénin méridional et central: indicateurs, scénarios et prospective de la sécurité alimentaire. Thèse de doctorat, EDP/FLASH/UAC, 302p.

Renard JF, Cheik L, Knips V. 2004. L'élevage et l'intégration régionale en Afrique de l'ouest. Ministère des Affaires Etrangères, FAO-CIRAD, 37p.

République du Sénégal. 2007. Caractérisation des systèmes de production agricole au Sénégal. Document synthèse, 39p.

SCDA (Secteur Communal pour le Développement Agricole)/Adjohoun). 2016. Données statistiques de la campagne agricole. Rapport final, 78 p.

Schwartz D. 1995. Méthodes Statistiques à l'Usage des Médecins et des Biologistes ( $4^{\mathrm{e}}$ edn). Editions médicales Flammarion : Paris ; 314.

Sènouvo P. 2000. Adaptation des fascines contre l'ensablement des trous à poissons « houédos » dans les conditions de plaines alluviales à Gangban (vallée de l'Ouémé) au Bénin. Rapport final - Volet Pêche et Pisciculture/R-D Sud/RAMR/INRAB, 16 p.
Sintondji LO, Agbossou EK, Degnissodé B. 2013. Dynamique de dégradation des forêts galeries et comblement du cours d'eau Agbado dans le département des Collines au Bénin. Int. J. Biol. Chem. Sci., 7(4): 1555-1567. DOI : http://dx.doi.org/10.4314/ijbcs.v7i4.12

Sogansa NY. 2014. Caractérisation des trous traditionnels à poissons du Haut delta de l'Ouémé au Bénin: Typologie, fonctionnement, Biodiversité et productivité. Mémoire de Master Recherche. Institut du Développement Rural. Université Polytechnique de Bobo-Dioulasso, 114p.

Son G, Kiogo R, Ye SG. 2016. Analyse des systèmes de production de l'oignon bulbe autour du barrage deGoinré dans la province du Yatenga au Nord du Burkina Faso. Int. J. Biol. Chem. Sci., 10(3). 1173-1183 DOI http://dx.doi.org/10.4314/ijbcs.v10i3.22

Terrier M, Gasselin P, Le Blanc J. 2010. Assessing the sustainability of activity systems to support agricultural households' projects. Methods and procedures for building sustainable farming systems, WS2.1, 11p.

Yoda FB. 2009. Sécurisation foncière en milieu rural au Burkina Faso. «L'accès à la terre et ses usages ». Rencontres lascaux, $5 \mathrm{p}$.

Zannou DV. 2017. Mise en valeur agricole et protection de l'environnement dans la basse de l'Ouémé au sud du Bénin : une étude de durabilité écologique. Thèse de doctorat en Géosciences de l'environnement et Aménagement de l'espace, Faculté des Lettres, Arts et Sciences Humaines, Université d'Abomey-Calavi, 291p. 